\title{
Fra-1 is upregulated in gastric cancer tissues and affects the PI3K/Akt and p53 signaling pathway in gastric cancer
}

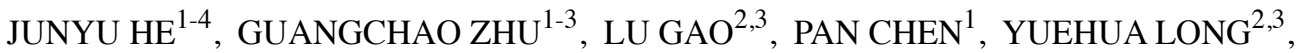

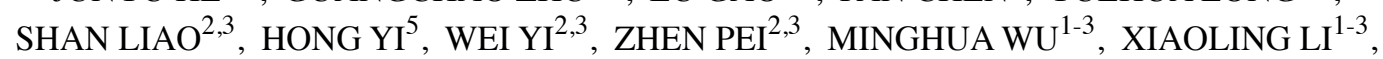 \\ JUANJUAN XIANG ${ }^{1-3}$, SHUPING PENG ${ }^{1-3}$, JIAN MA ${ }^{1-3}$, MING ZHOU $^{1-3}$, WEI XIONG ${ }^{1-3}$, ZHAOYANG ZENG $^{1-3}$, \\ BO XIANG ${ }^{1-3}, \mathrm{KE} \mathrm{TANG}^{2,3}, \mathrm{LI} \mathrm{CAO}^{2,3}$, GUIYUAN LI $^{1-3}$ and YANHONG ZHOU ${ }^{1-3}$ \\ ${ }^{1}$ Hunan Provincial Tumor Hospital and the Affiliated Tumor Hospital of Xiangya School of Medicine, Central South \\ University, Changsha, Hunan 410006; ${ }^{2}$ Cancer Research Institute, Central South University, Changsha, Hunan 410078; \\ ${ }^{3}$ Key Laboratory of Carcinogenesis of Ministry of Health and Key Laboratory of Carcinogenesis and Cancer Invasion \\ of Ministry of Education, Cancer Research Institute, Central South University, Changsha, Hunan 410078; \\ ${ }^{4}$ Department of Clinical Laboratory, Brain Hospital of Hunan Province; ${ }^{5}$ Research Center of Carcinogenesis \\ and Targeted Therapy, Xiangya Hospital, Central South University, Changsha, Hunan 410008, P.R. China
}

Received June 18, 2015; Accepted July 30, 2015

DOI: $10.3892 /$ ijo.2015.3146

\begin{abstract}
Gastric cancer is an aggressive disease that continues to have a daunting impact on global health. Fra-1 (FOSL1) plays important roles in oncogenesis in various malignancies. We investigated the expression of Fra-1 in gastric cancer (GC) tissues by qPCR, immunohistochemistry (IHC) and western blot technologies. The results showed that Fra-1 was overexpressed in gastric cancer tissues compared with the adjacent non-cancerous tissues. To explore the possible mechanism of Fra-1 in GC, we elucidated the effect of Fra-1 in the apoptosis and cell cycle of gastric cancer cells, AGS, and found that a considerable decrease in apoptotic cells and increase of $S$ phase rate were observed for AGS cells with Fra-1 overexpession. We identified and confirmed that Fra-1 affected the expression level of CTTN and EZR in vitro through LC-MS/MS analyses and western blot technology. Furthermore, we found that Fra-1 was correlated with dysregulation PI3K/Akt and p53 signaling pathway in gastric cancer tissues in vitro. Moreover, we found that Fra-1 overexpression affected the expression of PI3K, Akt, MDM2 and p53 in vivo. In summary, our results suggest
\end{abstract}

Correspondence to: Dr Yanhong Zhou or Professor Guiyuan Li, Cancer Research Institute, Central South University, No. 110 of Xiangya Road, Changsha, Hunan 410078, P.R. China

E-mail: zhouyanhong@csu.edu.cn

E-mail: lgy@csu.edu.cn

Abbreviations: GC, gastric cancer; FOSL1 (also known as Fra-1), FOS-like antigen 1; CTTN, cortactin; EZR, ezrin; TP53, tumor protein p53; AKT1 (also known as AKT), v-akt murine thymoma viral oncogene homolog 1; IHC, immunohistochemistry; GADPH, glyceraldehyde-3-phosphate dehydrogenase

Key words: Fra-1, gastric cancer, PI3K, p53, signal pathway that Fra-1 is upregulated in gastric cancer tissues and plays its function by affecting the PI3K/Akt and p53 signaling pathway in gastric cancer.

\section{Introduction}

Fra-1 is a member of the Fos transcription factor family (c-Fos, FosB, Fra-1 and Fra-2) that is highly expressed in multiple malignancy, and playing important roles in proliferation, transformation and metastasis (1,2). FRA-1 forms activator protein-1 complexes in association with members of the JUN family (c-Jun, JunB and JunD) and drives gene transcription $(1,2)$. Fra-1 can positively regulate transcription. Fra-1 activity is regulated transcriptionally as well as post-translationally $(3,4)$. FRA-1 has been implicated in the development of airway squamous metaplasia and is frequently overexpressed in squamous cell carcinomas of the stomach and esophagus (2). Fra-1 gene induction and the accumulation of Fra-1 protein might contribute to the neoplastic phenotype in HNSCC (head and neck squamous cell carcinoma) (3). Fra-1 is required for invasion and Fra-1 might be a good target for intervention in colorectal cancer $(3,5,6)$. In addition to its pro-invasive and pro-migratory effect, Fra-1 might influence the metastatic potential of breast cancer cells by changing the expression of adhesion molecules, resulting in increased adherence to endothelial cells $(1,7,8)$. Although some evidence has been reported, the mechanism of Fra-1 in malignancy is not fully understood. Moreover, at present, scarce information exists to study the relationship between Fra-1 and gastric. Thus, it is urgent to explore the effect and mechanism of Fra-1 in gastric cancer.

The PI3K (phosphoinositide 3-kinase)/Akt signaling pathway is a major driving force in a variety of cellular functions. Dysregulation of this pathway has been implicated in many human diseases including cancer (9-12). Therapy resistance is critical to tumor maintenance and severely limits 
Table I. Characteristics of gastric cancer patients.

\begin{tabular}{ccll}
\hline Samples & Age (years) & Gender & \multicolumn{1}{c}{ Histological diagnosis } \\
\hline 1 & 60 & Female & Gastric intermediately differentiated adenocarcinoma \\
2 & 60 & Male & Gastric poorly differentiated adenocarcinoma \\
3 & 46 & Male & Gastric intermediately differentiated adenocarcinoma \\
4 & 49 & Male & Gastric poorly differentiated adenocarcinoma \\
5 & 57 & Male & Gastric poorly differentiated adenocarcinoma \\
6 & 72 & Male & Gastric poorly differentiated adenocarcinoma \\
7 & 53 & Female & Gastric intermediately differentiated adenocarcinoma \\
8 & 63 & Male & Gastric poorly differentiated adenocarcinoma \\
9 & 54 & Female & Gastric poorly differentiated adenocarcinoma \\
10 & 66 & Male & Gastric poorly differentiated adenocarcinoma \\
11 & 57 & Male & Gastric poorly differentiated adenocarcinoma \\
12 & 47 & Male & Gastric poorly differentiated adenocarcinoma \\
13 & 70 & Female & Gastric intermediately differentiated adenocarcinoma \\
14 & 54 & Male & Gastric poorly differentiated adenocarcinoma \\
15 & 59 & Female & Gastric intermediately differentiated adenocarcinoma \\
16 & 62 & Male & Gastric poorly differentiated adenocarcinoma \\
17 & 60 & Male & Gastric poorly differentiated adenocarcinoma \\
18 & 73 & Male & Gastric poorly differentiated adenocarcinoma \\
19 & 47 & Female & Gastric poorly differentiated adenocarcinoma \\
20 & 63 & &
\end{tabular}

cancer patient survival. The PI3K pathway has emerged as a major driver of resistance to diverse anticancer agents (10). It has been shown that p53 was a crucial transcription factor and p53 is an important sensor of cellular stress under genotoxic, chemotoxic, pathological, and even normal physiological conditions $(13,14)$. MDM2 is a negative regulator of $\mathrm{p} 53$. Abnormalities in the p53 gene and overexpression of MDM2 are commonly observed in malignancy. The MDM2-p53 feedback loop plays an important role in tumor progression $(15,16)$. Mutations activating the PI3K/Akt signalling pathway and inactivating the p53 tumor suppressor gene are common mechanisms that cancer cells require to proliferate and escape pre-programmed cell death (17).

In the present study, we examined the expression levels of Fra-1 in gastric cancer tissues. Furthermore, we studied the influence of Fra-1 to gastric cancer and explored the possible mechanism.

\section{Materials and methods}

Cell culture. AGS, a human gastric cancer cell line was cultured in HAM'S/F-12 (HyClone Laboratories, Logan, UT, USA) supplemented with $10 \%$ fetal bovine serum (FBS; Gibco by Life Technologies ${ }^{\mathrm{TM}}$, Grand Island, NY, USA), $100 \mathrm{U} / \mathrm{ml}$ penicillin and $100 \mu \mathrm{g} / \mathrm{ml}$ streptomycin (GE Healthcare Life Sciences, Logan, UT, USA) at $37^{\circ} \mathrm{C}$ in the presence of $5 \% \mathrm{CO}_{2}$.

Patient samples. Twenty participants were recruited at the Cancer Hospital of Hunan province, Central South University (Changsha, Hunan, China). Consent forms were obtained from each individual patient, and experimental protocols were approved by the Institutional Review Board of the Cancer Hospital of Hunan province. All subjects enrolled in the study were Chinese. All clinical and biological data were available for the samples (Table I). Gastric cancer tissue and corresponding non-tumor normal tissue were collected, and each biopsy sample was divided into two sections, one was submitted to routine histological diagnosis, and the remaining section was studied by qPCR, immunohistochemistry and western blot experiments.

Total RNA extraction and quantitative real-time PCR analysis. Total RNA was extracted from gastric cancer tissues and corresponding non-tumor normal tissues using TRIzol reagent (CWBio, Beijing, China) and cDNA synthesis was carried out using the RevertAid First Strand cDNA Synthesis kit (CWBio) according to the manufacturer's recommendations. Quantitative real-time PCR (qRT-PCR) was done with GoTaq ${ }^{\circledR}$ qPCR Master Mix (Promega Corp., Fitchburg, WI, USA). For detection of Fra-1 mRNA expression levels, GAPDH was amplified in parallel as an internal control. The sequences of the primers used for qPCR were as follows: Fra-1 forward, 5'-gcatgggctaaggatttgaa-3' and reverse, 5'-tcccaaatttagcetgtt gg-3'; GAPDH forward, 5'-cgaccactttgtcaagctca-3' and reverse, 5'-actgagtgtggcagggactc-3'. The expression of mRNA was assessed by evaluated threshold cycle (CT) values. The CT values were normalized with the expression levels of GAPDH and the relative amount of mRNA specific to each of the target genes was calculated using the $2^{-\Delta \Delta C T}$ method $(4,18-21)$. qPCR was done with the Bio-Rad CFK96 ${ }^{\mathrm{TM}}$ Real-Time System (BioRad Laboratories, Hercules, CA, USA). The data were analyzed by Bio-Rad CFK manager software (Bio-Rad Laboratories). 
Expression of mRNA was assessed by evaluated threshold cycle (CT) values, and GAPDH was used as an internal control.

Immunohistochemistry (IHC) and evaluation of staining. Immunohistochemistry was done using the peroxidase antiperoxidase technique following a microwave antigen retrieval procedure. Antibody for $\mathrm{Fra}-\mathrm{l}$ was purchased from Wuhan Boster Biological Technology, Ltd. (Wuhan, China). Antibody against Fra-1 (1:100) was overlaid on gastric cancer and corresponding non-tumor normal tissue sections and incubated overnight at $4^{\circ} \mathrm{C}$. Secondary antibody incubation (Santa Cruz Biotechnology, Inc., Santa Cruz, CA, USA) was performed at room temperature for $30 \mathrm{~min}$.

Sections were blindly evaluated by two investigators in an effort to provide a consensus on staining patterns by light microscopy (Olympus). Fra-1 staining was assessed according to the methods described by Hara and Okayasu (22) with minor modifications. Each case was rated according to a score that added a scale of intensity of staining to the area of staining. At least 10 high-power fields were chosen randomly, and $>1,000$ cells were counted for each section. The intensity of staining was graded on the following scale: 0 , no staining; $1+$, mild staining; $2+$, moderate staining; $3+$, intense staining. The area of staining was evaluated as follows: 0 , no staining of cells in any microscopic fields; $1+,<30 \%$ of tissue stained positive; $2+$, between 30 and $60 \%$ stained positive; $3+,>60 \%$ stained positive. The minimum score when summed (extension + intensity) was, therefore, 0 , and the maximum, 6 . A combined staining score (extension + intensity) of $\leq 2$ was considered to be negative (low staining); a score between 3 and 4 was considered to be moderate; whereas, a score between 5 and 6 was considered to be strong.

Construction of pEGFP-N1-Fra-1 vector and cell transfection. The coding region of Fra-1 gene was generated by PCR with the primer pair $5^{\prime}$-atactcgaatgaacctggccatcagcat-3' and 5 '-gcggaattctcacagggacatgaaatccg-3'. The PCR was performed under the following conditions conditions: one cycle for $5 \mathrm{~min}$ at $94^{\circ} \mathrm{C} ; 30$ cycles for $45 \mathrm{sec}$ at $94^{\circ} \mathrm{C}, 45 \mathrm{sec}$ at $55^{\circ} \mathrm{C}$, and $90 \mathrm{sec}$ at $72^{\circ} \mathrm{C}$, and extension of $10 \mathrm{~min}$ at $72^{\circ} \mathrm{C}$. The fragments were cloned into the TA vector (Promega) and used to transform E. coli JM109 (Takara Bio, Dalian, China). Following selection and propagation, the pure plasmid DNA was prepared by standard methods. The DNA fragments were removed from the TA vector by restriction enzyme digestion with XhoI and $E c o$ R1 (Promega) to subclone into the pEGFP-N1 vector. The fusion sequences were verified by DNA sequencing using ABI 3730.

Cell transfection. Cell transfection was accomplished by using Lipofectamine, according to the manufacturer's instruction (Invitrogen, Carlsbad, CA, USA). A $2 \times 10^{5}$ cells were planted into each well of a 6 -well plate $24 \mathrm{~h}$ prior to the transfection. For each transfection, $2 \mu \mathrm{g}$ of pEGFP-N1-Fra-1 plasmid and pEGFP-N1 vector plasmid was transfected into AGS cells, respectively. The plasmids were diluted with $100 \mu 1$ of serumfree media and $4 \mu \mathrm{l}$ Lipofectamine was added into $100 \mu \mathrm{l}$ serum-free media. The two solutions were combined, mixed gently and incubated at room temperature for $30 \mathrm{~min}$. Then the $200 \mu 1$ mixture and $200 \mu 1$ of serum-free media were added into each well. The cells were then incubated at $37^{\circ} \mathrm{C}$ for $24 \mathrm{~h}$, followed by replacing the transfection media with fresh complete culture media. After an additional 24-h culture, the cells were harvested for the following western blot experiments.

Flow cytometric analysis of cell cycle. The cell cycle was analyzed by flow cytometric analysis using a Moflo ${ }^{\mathrm{TM}}$ XDP high-performance cell sorter (Beckman Coulter, Brea, CA, USA) propidium iodide (PI) (Nanjing KeyGen Biotech Co., Ltd., Nanjing, China). Briefly, cells $\left(2 \times 10^{5}\right)$ were placed in each well of a 6 -well plate $24 \mathrm{~h}$ prior to the transfection. For each transfection, $2 \mu \mathrm{g}$ of pEGFP-N1-Fra-1 plasmid and pEGFP-N1 vector plasmid was transfected into AGS cells, respectively. The plasmids were diluted with $100 \mu \mathrm{l}$ of serum-free media and $4 \mu \mathrm{l}$ Lipofectamine was added into $100 \mu \mathrm{l}$ serum-free media. The two solutions were combined, mixed gently and incubated at room temperature for $30 \mathrm{~min}$. The $200 \mu \mathrm{l}$ mixture and $200 \mu \mathrm{l}$ of serum-free media were added into each well. The cells were then incubated at $37^{\circ} \mathrm{C}$ for $24 \mathrm{~h}$, followed by replacing the transfection media with fresh complete culture media. After an additional 24-h culture, the cells were harvested for cell apoptosis analysis. The cells were harvested and fixed in $70 \%$ ethanol at $4^{\circ} \mathrm{C}$. Subsequently, the cells were washed with cold PBS and stained with propidium iodide (PI) in working solution $(0.5 \mathrm{mg} / \mathrm{ml} \mathrm{RNase}$, and $0.1 \mathrm{mg} / \mathrm{ml}$ propidium iodide in PBS). The cell cycle was characterized by flow cytometric analysis using a MoFlo ${ }^{\mathrm{TM}}$ XDP high-performance cell sorter (Beckman Coulter) and the data were analyzed by the summit 5.2 software (Beckman Coulter).

The effect of Fra-1 to gastric cancer cell apoptosis. The cell apoptosis was analyzed by flow cytometric analysis using a $\mathrm{MoFlo}^{\mathrm{TM}}$ XDP high-performance cell sorter (Beckman Coulter) propidium iodide (PI) and Hoechst 33342 double staining (Nanjing KeyGen). Briefly, cells $\left(2 \times 10^{5}\right)$ were placed in each well of a 6-well plate $24 \mathrm{~h}$ prior to the transfection. For each transfection, $2 \mu \mathrm{g}$ of pEGFP-N1-Fra-1 plasmid and pEGFP-N1 vector plasmid was transfected into AGS cells, respectively. The plasmids were diluted with $100 \mu \mathrm{l}$ of serumfree media and $4 \mu \mathrm{l}$ Lipofectamine was added into $100 \mu \mathrm{l}$ serum-free media. The two solutions were combined, mixed gently and incubated at room temperature for $30 \mathrm{~min}$. The $200 \mu \mathrm{l}$ mixture and $200 \mu \mathrm{l}$ of serum-free media were added into each well. The cells were then incubated at $37^{\circ} \mathrm{C}$ for $24 \mathrm{~h}$, followed by replacing the transfection media with fresh complete culture media. After an additional 24-h culture, the cells were harvested for cell apoptosis analysis. Cells were collected in an Eppendorf tube for $24 \mathrm{~h}$ and washed twice with PBS by centrifugation. The supernatants were discarded. To detect apoptosis, $500 \mu$ l PBS, $5 \mu 1$ Hoechst 33342 and $5 \mu 1$ PI were added to each tube, and the contents of the tube were mixed in the dark, at room temperature for $15 \mathrm{~min}$, followed by FCM testing (Beckman Coulter). The data acquired were analyzed with summit v5.2 software (Beckman Coulter).

Identification of differential proteins of Fra-1 overexpressing AGS cells by LC-MS/MS analysis. The cell lysate consisted of $50 \mathrm{mM}$ Tris (pH 7.4), $150 \mathrm{mM} \mathrm{NaCl}, 1 \%$ Triton X-100, $1 \%$ sodium deoxycholate, $0.1 \%$ SDS and sodium orthovanadate, sodium fluoride, EDTA, leupeptin, supplemented with $1 \mathrm{X}$ halt 
protease inhibitor cocktail (CWBio) and $1 \mathrm{X}$ halt phosphatase inhibitor cocktail (BestBio). The protein concentration was estimated by bicinchoninic acid (BCA) method. The gel was run at $80 \mathrm{~V} 40 \mathrm{~min}$, then $120 \mathrm{~V} 90 \mathrm{~min}$. Then, $50 \mu \mathrm{g}$ of each preparation was loaded on a $10 \%$ SDS-PAGE gel. SDS-PAGE was run at $80 \mathrm{~V} 40 \mathrm{~min}$, then $120 \mathrm{~V} 90 \mathrm{~min}$. Protein bands were visualized using Coomassie brilliant blue G-250 (Sigma, Carlsbad, CA, USA) and excised. The protein spots were destained using $15 \mathrm{mM} \mathrm{K} \mathrm{K}_{4} \mathrm{Fe}(\mathrm{CN})_{6}$ and $50 \mathrm{mM}$ sodium thiosulfate $1.25 \mu \mathrm{g}$ trypsin (1:20 enzyme/substrate ratio) was added to each band and in-gel-digestion was performed at $37^{\circ} \mathrm{C}$ overnight $(\sim 16 \mathrm{~h})$. The generated peptides were extracted by sonication (15 min, ice cooling) of the gel pieces in $\sim 20 \mu \mathrm{l}$ of $50 \%$ acetonitrile in $0.1 \% \mathrm{FA}$, twice. After extracting from gel pieces, peptides were dried by vacuum centrifugation to ensure a complete removal of acetonitrile and reconstituted in $20 \mu 10.1 \%$ FA (23-25).

LC-MS/MS analyses were performed on an Ultimate $^{\mathrm{TM}}$ 3000 RSLCnano system online coupled to an LTQ Orbitrap Velos Pro mass spectrometer (both from Thermo Scientific, Bremen, Germany). Peptide diluted with $0.1 \%$ FA, was injected in each analysis of $30 \mu 1$ sample. After injection, peptides were pre-concentrated with $0.1 \% \mathrm{FA}, 3 \% \mathrm{ACN}$ on a trap column ( $\mu$-Precolumn C18 PepMap 100, $300 \mu \mathrm{m} \times 5 \mathrm{~mm}, 5 \mu \mathrm{m}$, $100 \AA$ A Thermo Fisher Scientific) at a flow rate of $300 \mathrm{nl} / \mathrm{min}$ for $5 \mathrm{~min}$. Subsequently, the analyte was transferred to the analytical column (Acclaim ${ }^{\circledR}$ PepMap RSLC, $75 \mu \mathrm{m}$ x $15 \mathrm{~cm}$, nano Viper, C18, $2 \mu \mathrm{m}, 100 \AA$; Thermo Fisher Scientific) and separated using a 120 -min gradient from 5 to $40 \%$ solvent B at a flow rate of $300 \mathrm{nl} / \mathrm{min}$ (solvent $\mathrm{A}, 0.1 \%$ formic acid; solvent B, $0.08 \%$ FA $80 \%$ acetonitrile). The mass spectrometer was operated in a data-dependent mode. The general mass spectrometric parameters were: spray voltage, $2.0 \mathrm{kV}$; capillary temperature, $275^{\circ} \mathrm{C}$. For data-dependent MS/MS analyses, the software Xcalibur (Thermo Fisher Scientific) was used. Full scan MS spectra were acquired at a mass resolution of 60,000 (mass range 350-2000 m/z) in the Orbitrap analyzer. For labelfree analyses, tandem mass spectra of the ten most abundant peaks were acquired in the linear ion trap by peptide fragmentation using collision-induced dissociation (CID). Normalized collision energy (NCE) was set to $35 \%$ and an isolation width of $2 \mathrm{~m} / \mathrm{z}$ was chosen (23-25).

Protein identifications were performed with Proteome Discoverer software. Briefly, Thermo raw-files were imported and searched against UniProtKB/Swiss-Prot database (release 2014_10). For database searches, mass tolerances were set to $10 \mathrm{ppm}$ and $0.8 \mathrm{Da}$ for precursor and fragment ions, respectively. Taxonomy was restricted to human and one enzymatic miscleavage was allowed. For label-free analyses, modifications of cysteine (carbamidomethyl, static) and methionine (oxidation, variable) were considered. Confidence of peptide identifications was estimated using percolator function, implemented in Proteome Discoverer. Instead of determining the peptide confidence based on a singlemetric such as Mascot ion score, we decided to use percolator as it discriminates correct from incorrect peptide spectrum matches based on multiple orthogonal score criteria leading to accurate and sensitive peptide identifications. Peptide identifications with false discovery rates $\mathrm{N} 1 \%$ (q-value N 0.01) were discarded (23-25).
Western blot analysis. The gastric cancer tissues, corresponding non-tumor normal tissues, and AGS cells were lysed in RIPA buffer (CWBio) and total protein concentration was determined using Pierce ${ }^{\circledR}$ BCA protein assay kit (Thermo Scientific, Inc., Rockford, IL, USA). Extracts containing $50 \mu \mathrm{g}$ of proteins were separated in $10 \%$ SDS-PAGE gels and electroblotted onto nitrocellulose membranes (HyClone Laboratories). The membranes were inhibited using Trisbuffered saline/Tween-20 (25 mM Tris- $\mathrm{HCl}, 150 \mathrm{mM} \mathrm{NaCl}$, $\mathrm{pH} 7.5$ and $0.05 \%$ Tween-20) containing 5\% non-fat milk followed by overnight incubation at $4{ }^{\circ} \mathrm{C}$ with primary antibodies (rabbit anti-PI3K polyclonal antibody, catalog no. 4292; Cell Signaling Technology, Inc., Danvers, MA, USA; dilution, 1:500; rabbit anti-Akt polyclonal antibody, catalog no. 9272, Cell Signaling Technology; dilution, 1:300; rabbit antiMDM2 antibody, 1:200 and rabbit anti-p53 antibody, 1:200; Wuhan Boster). Following three washes, the membranes were incubated with horseradish peroxidase-conjugated second antibodies (catalog no. sc-2491; Santa Cruz Biotechnology, Inc., Santa Cruz, CA, USA; dilution, 1:5,000) and the specific signals were visualized using an ECL detection system. AntiGAPDH antibody (Santa Cruz Biotechnology; 1:3,000) was used as a loading control.

Statistical analysis. Differences of non-parametric variables were analyzed by the Mann-Whitney U test. Differences of the quantitative variables between groups were analyzed by the Student's t-test using SPSS 11.0 program (SPSS, Inc., Chicago, IL, USA). A value of $\mathrm{P}<0.05$ was considered statistically significant.

\section{Results}

Fra-1 is highly expressed in gastric cancer tissue. To detect the mRNA expression levels of the Fra-1 molecular in gastric cancer and the adjacent non-cancerous tissues, 20 samples of each were selected to perform qPCR of the Fra-1 gene. The data were analyzed using the $2^{-\Delta \Delta C T}$ method and the fold change in the expression of these genes relative to the internal control gene, GAPDH, was analyzed. The expression of the Fra-1 gene was higher in the gastric cancer samples compared with the adjacent non-cancerous tissues and the normalized Fra-1 gene expression in gastric cancer was upregulated 2.99fold $(\mathrm{P}=0.017)$ (Fig. 1A and Table II).

To determine whether the Fra-1 gene was expressed at a higher level in gastric cancer compared with the adjacent non-cancerous tissues, the protein expression levels of Fra-1 were further examined by western blot analysis in 1-5 samples (Fig. 1B). In comparison with the adjacent non-cancerous tissues, the expression level was identified to be greater in gastric cancer tissues, which corresponded with the qPCR results.

To confirm the pattern of Fra-1 in gastric cancer, immunohistochemistry (IHC) was carried out with antibodies against Fra-1 protein in gastric cancer and the adjacent non-cancerous tissues. Fra-1 was identified as differentially expressed between gastric cancer tissues versus the adjacent non-cancerous tissues. IHC showed a similar pattern in protein expression with western blot results. There was $61.5 \%$ (16/26) high score of Fra-1 in gastric cancer tissues and $26.9 \%(7 / 26)$ in the adjacent non-cancerous tissues. The distribution of low score was $23.1 \%(6 / 26)$ and 
Table II. Identification of the mRNA expression level of Fra-1 in gastric cancer and adjacent non-cancerous tissues by qPCR.

\begin{tabular}{|c|c|c|c|c|c|c|c|}
\hline Gene & Sample & $\mathrm{n}$ & $\begin{array}{c}\text { Fra-1 CT } \\
(\text { mean } \pm \mathrm{SD})\end{array}$ & $\begin{array}{l}\text { GAPDH CT } \\
(\text { mean } \pm \text { SD })\end{array}$ & $\begin{array}{c}\Delta \mathrm{CT} \\
(\text { mean } \pm \mathrm{SD})\end{array}$ & $\begin{array}{c}\Delta \Delta \mathrm{CT} \\
(\text { mean } \pm \mathrm{SD})\end{array}$ & Fold $^{\mathrm{a}}$ \\
\hline \multirow[t]{2}{*}{ Fra-1 } & Gastric cancer tissues & 20 & $26.86 \pm 1.05$ & $16.27 \pm 0.64$ & $10.59 \pm 0.43$ & $-1.58 \pm 0.52$ & 2.99 \\
\hline & Non-cancerous tissues & 20 & $29.34 \pm 1.65$ & $16.73 \pm 0.66$ & $12.17 \pm 0.45$ & & $(2.08-4.28)$ \\
\hline
\end{tabular}

${ }^{\mathrm{a}}$ Mean fold change in expression of the target gene, Fra-1, relative to the internal control gene, GAPDH, was calculated using the $2^{-\Delta \Delta \mathrm{CT}}$ equation by Livak et al (18): $\Delta \Delta \mathrm{CT}=\left(\mathrm{CT}_{\text {Target }}-\mathrm{CT}_{\mathrm{GAPDH}}\right)$ cervical cancer - $\left(\mathrm{CT}_{\text {Target }}-\mathrm{CT}_{\mathrm{GAPDH}}\right)$ control. At least three replicates of each reaction were performed. $\mathrm{CT}$, threshold cycle; qPCR, quantitative polymerase chain reaction.

Table III. The difference of Fra-1 expression between gastric cancer and the adjacent non-cancerous tissues by immunohistochemistry.

\begin{tabular}{lrrrrrr}
\hline & & \multicolumn{4}{c}{ Score } \\
\cline { 3 - 6 } & $\mathrm{n}$ & Low (0-2) & Moderate (3-4) & High (5-6) & $\chi^{2}$ & P-value \\
\hline Gastric cancer & 30 & $6(20.0 \%)$ & $8(26.7 \%)$ & $16(61.5 \%)$ & 7.38 & $0.024<0.05$ \\
Non-cancerous tissues & 30 & $15(42.3 \%)$ & $8(30.8 \%)$ & $7(26.9 \%)$ & &
\end{tabular}

A

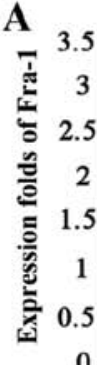

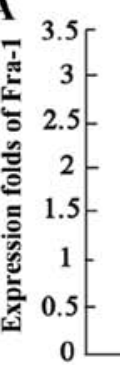

B

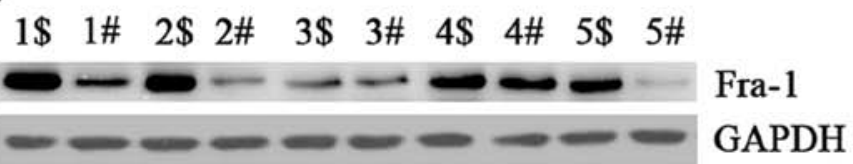

Group
C
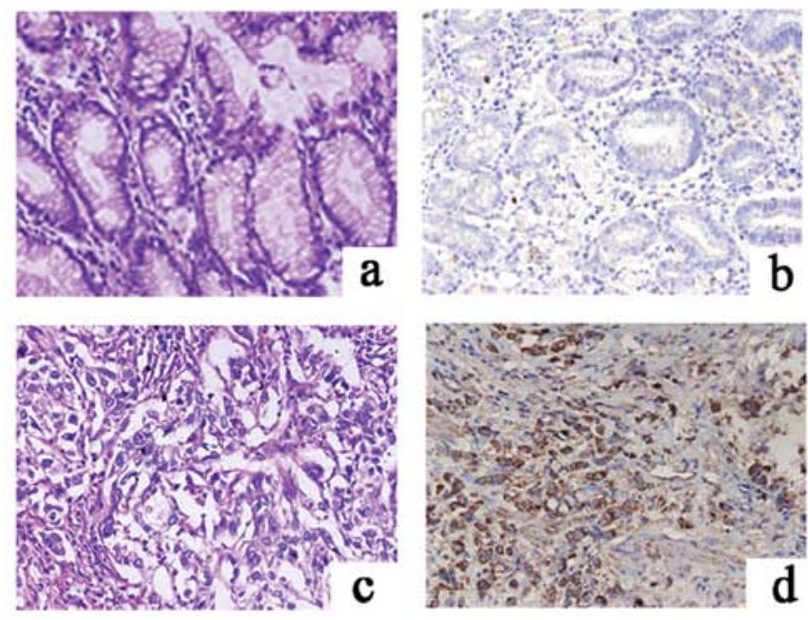

Figure 1. The detection of expression levels of FRA-1 in the gastric cancer tissues and the adjacent non-cancerous tissues by qPCR, western blot analysis and IHC. (A) qPCR was performed to validate the expression of FRA-1 in gastric cancer tissues and the adjacent non-cancerous tissues. GAPDH was used as an internal control and for normalization of the data. The mRNA expression levels of the FRA-1 was significantly upregulated in the gastric cancer tissues ( $\mathrm{n}=20$ ) compared with the adjacent non-cancerous tissues $(n=20)$. ${ }^{*} \mathrm{P}<0.01$. (B) $1,2,3,4$ and 5 tissues which were used in the detection of mRNA expression levels by qPCR were selected to detect the expression levels of FRA-1 protein by western blot analysis. \$ indicates gastric cancer specimens and \# indicates adjacent non-cancerous tissues. Data are representative of three independent experiments. (C) Immunohistochemistry analysis of the expression of FRA-1 protein in the gastric cancer and the adjacent non-cancerous tissues. Antibody of FRA-1 protein was used; brown grains denote positive signal. (a) H\&E staining of gastric epithelial tissue, (b) FRA-1 staining of gastric epithelial tissue, (c) H\&E staining of gastric cancer tissue, (d) FRA-1 staining of gastric cancer tissue. Original magnification, $\mathrm{x} 200$.

$42.3 \%(11 / 26)$ in gastric cancer and the adjacent non-cancerous tissues, respectively $(\mathrm{P}=0.024)$ (Fig. $1 \mathrm{C}$ and Table III), thus, corresponding with the $\mathrm{qPCR}$ results.

Analysis of cell cycle in AGS cells overexpressing Fra-1. Cell cycle progression was assessed in AGS, AGS/vector and AGS/ Fra- 1 cells by flow cytometry. For AGS and AGS/vector cells, the percentages of $\mathrm{G} 0 / \mathrm{G} 1, \mathrm{~S}$ and $\mathrm{G} 2 / \mathrm{M}$ phases were 59.2, 22.4, 18.4 and 60.1, 22.0, 17.9\%, respectively. Whereas, AGS cells with Fra-1 overexpression were mostly in the proliferative phase, and showed significant increase in $\mathrm{S}$ phase and reduction in G0/G1 phase, the percentages of G0/G1, S and G2/M phases were 48.9, 32.4 and $18.7 \%$, respectively (Fig. 2A). The results suggested that Fra-1 affected cell cycle distribution of gastric cancer cells.

Fra-1 inhibits gastric cancer cell apoptosis. Promotion of cell proliferation is usually mediated by inhibition of cell apop- 


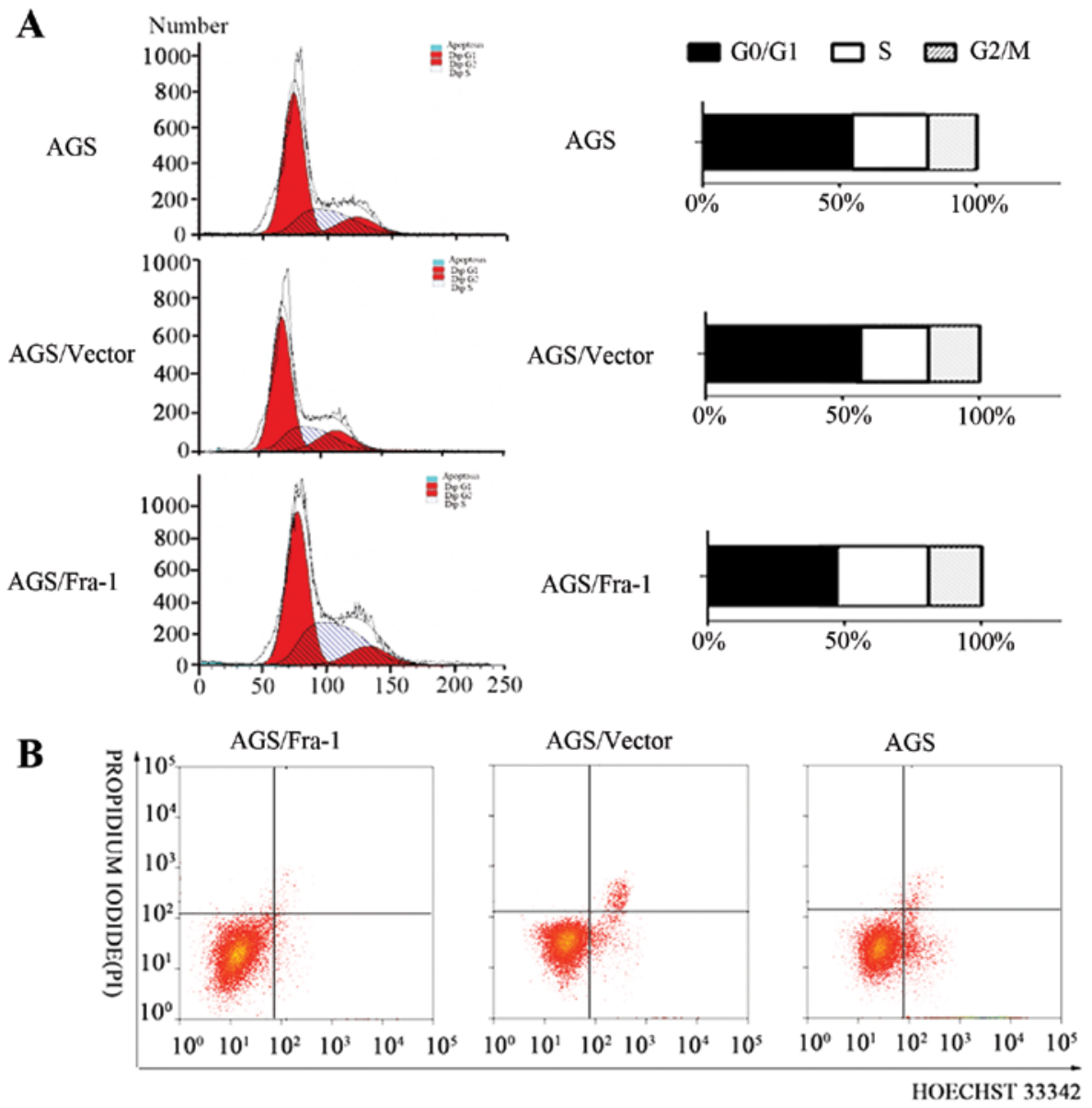

Figure 2. Fra-1 affects the distribution of cell cycle and inhibits cell apoptosis in AGS gastric cancer cells. (A) The distribution of the cell cycle in AGS gastric cancer cells. AGS/Fra-1, transfection with pEGFP-N1-Fra-1 plasmid after $48 \mathrm{~h}$, AGS/vector, transfection with pEGFP-N1 plasmid after $48 \mathrm{~h}$, AGS, transfection without plasmid. (B) The effect of Fra-1 to AGS gastric cancer cells. AGS/Fra-1, transfection with pEGFP-N1-Fra-1 plasmid after 48 h, AGS/ vector, transfection with pEGFP-N1 plasmid after 48 h, AGS, transfection without plasmid. Data are representative of three independent experiments.

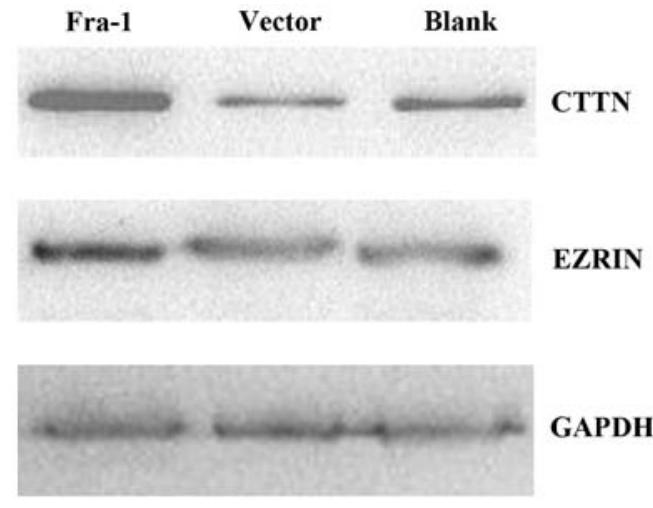

Figure 3. Expression levels of CTTN and EZR protein in AGS gastric cancer cells. Fra-1, AGS cells transfected with pEGFP-N1- Fra-1. Vector, AGS cells transfected with pEGFP-N1. Blank, AGS cells not transfected with plasmid. Data are representative of three independent experiments.

tosis. To determine whether apoptosis mediated the growth in AGS, AGS/vector and AGS/Fra-1 cells, we performed a Hoechst 33342/PI double-staining experiment. A considerable decrease in apoptotic cells was observed for AGS/Fra-1 cells $(5.27 \pm 0.24 \%)$, AGS cells $(11.54 \pm 0.87 \%)$, and AGS/vector cells $(10.97 \pm 0.73 \%$ ) (Fig. 2B). Our results suggested that Fra-1 inhibited gastric cancer cell apoptosis.

Identification of proteins affected by Fra-1 through LC-MS/ $M S$ analyses. We found that Fra-1 was highly expressed in gastric cancer tissues and inhibited apoptosis of gastric cancer cells in vitro. Furthermore, we identified the differential molecule affected by Fra-1 through LC-MS/MS analyses. Our results showed that there were 21 proteins which were only present in AGS/Fra-1 cells and there were 16 proteins which were only at present in AGS/vector cells. According to the frequency of unique peptides, the CARS protein was the highest in AGS/Fra-1 cells followed by CLTB, CTTN, EHD1, EPS8L2, and EZR (Table III). ACTG1 protein was the highest in AGS/vector cells (Table IV).

To confirm the reliability of the proteins identified, we tested the expression levels of CARS, CLTB, CTTN, EHD1, EPS8L2 and EZR by western blot analysis. The results showed that CTTN and EZR were upregulated in AGS/Fra-1 cells compared with AGS/vector cells and AGS cells (Fig. 3). It corresponded with the LC-MS/MS results. Further four proteins were no significantly different among AGS/Fra-1 cells, AGS/vector cells and AGS cells. 
Table IV. The differential proteins affected by FRA-1 through LC-MS/MS analyses.

\begin{tabular}{|c|c|c|c|c|c|c|c|c|}
\hline \multirow[b]{2}{*}{ No. } & \multicolumn{4}{|c|}{ AGS/FRA-1 } & \multicolumn{4}{|c|}{ AGS/vector } \\
\hline & Protein & $\begin{array}{l}\text { Unique } \\
\text { peptides }\end{array}$ & $\begin{array}{c}\text { Coverage } \\
(\%)\end{array}$ & $\begin{array}{l}\text { Protrin } \\
\text { score }\end{array}$ & Protein & $\begin{array}{l}\text { Unique } \\
\text { peptides }\end{array}$ & $\begin{array}{c}\text { Coverage } \\
(\%)\end{array}$ & $\begin{array}{c}\text { Protrin } \\
\text { score }\end{array}$ \\
\hline 1 & CARS & 48 & 25.05 & 262.99 & ACTG1 & 44 & 23.05 & 231.28 \\
\hline 2 & CLTB & 33 & 21.63 & 171.87 & ARF6 & 42 & 11.23 & 224.82 \\
\hline 3 & CTTN & 33 & 19.64 & 132.1 & CSTB & 30 & 24.68 & 150.08 \\
\hline 4 & EHD1 & 32 & 9.13 & 114.32 & DDX56 & 29 & 23.47 & 156.81 \\
\hline 5 & EPS8L2 & 31 & 24.33 & 179.56 & EIF4A1 & 28 & 43.83 & 119.16 \\
\hline 6 & EZR & 31 & 25.90 & 157.76 & LAMB3 & 26 & 16.73 & 137.39 \\
\hline 7 & GIPC1 & 30 & 46.99 & 149.72 & MTA2 & 26 & 16.30 & 72.34 \\
\hline 8 & GPRC5A & 27 & 20.13 & 144.92 & MYOF & 25 & 44.80 & 153.84 \\
\hline 9 & HMBS & 26 & 45.72 & 184.4 & PPM1G & 25 & 18.73 & 110.75 \\
\hline 10 & ITGA3 & 26 & 14.31 & 158.29 & RRP9 & 24 & 28.79 & 121.47 \\
\hline 11 & KRT17 & 24 & 48.59 & 151.03 & S100A16 & 23 & 47.65 & 168.64 \\
\hline 12 & LMO7 & 23 & 17.38 & 132.3 & TBRG4 & 23 & 32.13 & 96.54 \\
\hline 13 & LRRFIP1 & 21 & 26.11 & 104.45 & TM4SF1 & 21 & 37.00 & 175.58 \\
\hline 14 & MRPL17 & 20 & 36.47 & 158.23 & TOR4A & 21 & 51.35 & 167.67 \\
\hline 15 & NOP16 & 20 & 46.78 & 124.36 & TUBA1C & 21 & 12.18 & 102.87 \\
\hline 16 & NUDT5 & 20 & 29.51 & 105.72 & UBC & 20 & 39.47 & 157.66 \\
\hline 17 & RALB & 20 & 17.95 & 101.27 & & & & \\
\hline 18 & TTLL12 & 19 & 39.36 & 216.67 & & & & \\
\hline 19 & TUBB4B & 19 & 48.45 & 135.76 & & & & \\
\hline 20 & TUBG1 & 19 & 35.49 & 117.77 & & & & \\
\hline 21 & VPS26A & 19 & 32.71 & 95.42 & & & & \\
\hline
\end{tabular}

Ranked by unique peptides.

$1 \$ \quad 1 \# \quad 2 \$ \quad 2 \# \quad 3 \$ 3 \# \quad 4 \$ \quad 4 \#$

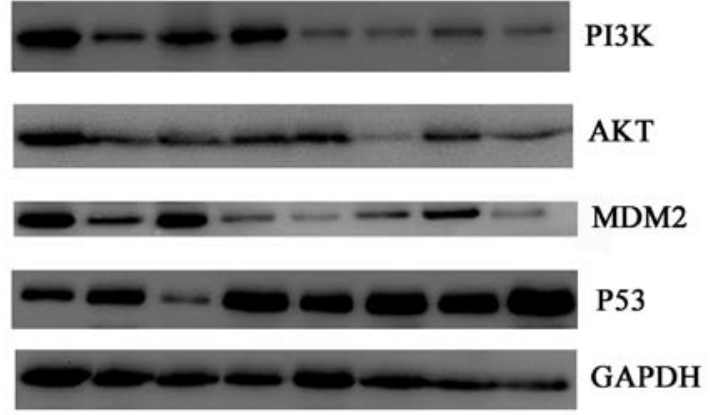

Figure 4. Expression levels of PI3K, AKT, p53 and MDM2 protein in gastric cancer and the adjacent non-cancerous tissues. In total, 1, 2, 3 and 4 tissues which were used in the detection of mRNA expression levels by qPCR were selected to detect the expression levels of PI3K, AKT, p53 and MDM2 protein by western blot analysis. $\$$ indicates gastric cancer specimens and \# indicates adjacent non-cancerous tissues. Data are representative of three independent experiments.

Fra-1 is correlated with dysregulation of PI3K, Akt, MDM2 and p53 in gastric cancer tissues in vitro. To uncover the possible mechanism of Fra-1 in gastric cancer, we tested the expression levels of key molecules in the PI3K/Akt signaling pathway by western blot technology. PI3K and Akt were upregulated in gastric cancer compared with the adjacent

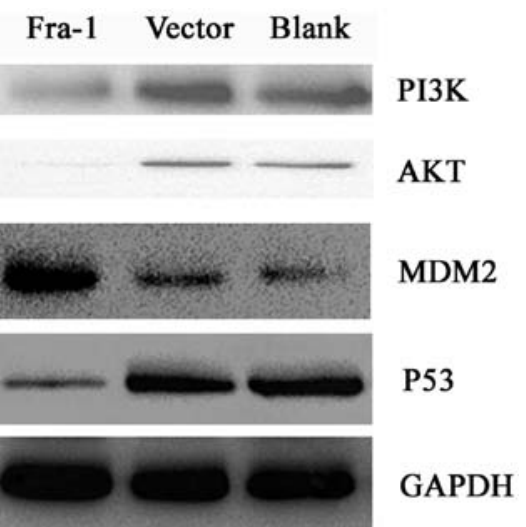

Figure 5. Expression levels of PI3K, AKT, p53 and MDM2 protein in AGS gastric cancer cell lines. Fra-1, AGS cells transfected with pEGFP-N1-Fra-1. Vector, AGS cells transfected with pEGFP-N1. Blank, AGS cells not transfected with plasmid. Data are representative of three independent experiments.

non-cancerous tissues. MDM2 had the same tendency with PI3K and Akt, but p53 was downregulated in gastric cancer (Fig. 4). Combined with the above results in which Fra-1 was highly expressed in gastric cancer, we inferred that Fra-1 is correlated with dysregulation PI3K/Akt and p53 signaling pathway in gastric cancer tissues in vitro. 


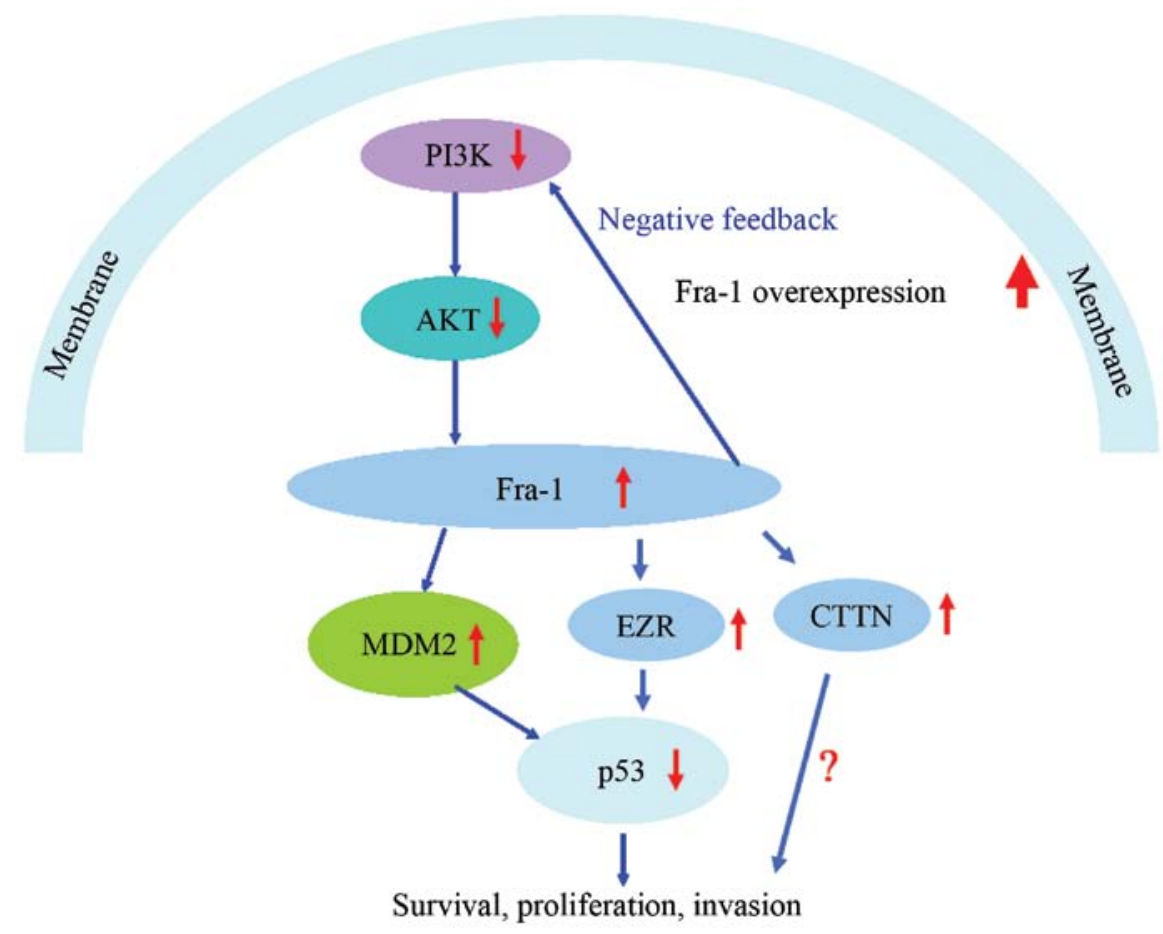

Figure 6. The proposed Fra-1 related pathway.

Fra-1 overexpression affects the expression of PI3K, Akt, MDM2 and p53 in vivo. To confirm whether Fra-1 affects the expression of PI3K, Akt, MDM2 and p53 in vivo, we constructed the plasmid pEGFP-N1-Fra-1. The plasmid of pEGFP-N1-Fra-1 and pEGFP-N1 were transfected into AGS gastric cancer cells. We harvested the cells after $48 \mathrm{~h}$ with transfection and tested the expression levels of PI3K, Akt, MDM2 and p53 proteins in vivo. The PI3K and Akt were downregulated in AGS cells which Fra-1 was overexpressed. The MDM2 was upregulated in AGS cells in which Fra-1 was overexpressed. Furthermore, p53 was downregulated in AGS cells in which Fra-1 was overexpressed (Fig. 5). Our results suggested that Fra-1 overexpression affected the expression of PI3K, Akt, MDM2 and p53 in vivo (summary in Fig. 6).

\section{Discussion}

Despite an overall decline in incidence over the last several decades, gastric cancer (GC) remains the fourth most common type of cancer and is the second leading cause of cancerrelated death worldwide $(26,27)$. Although some evidence has been reported, the mechanism of GC is not fully understood (28-30). Thus, identifying molecular aberrations in GC may improve our understanding of gastric carcinogenesis and help us subdivide patients into biologically and clinically relevant subgroups, as well as develop novel therapeutic strategies.

In the present study, we found that the expression of the Fra-1 gene was higher in the gastric cancer samples compared with the adjacent non-cancerous tissues and the normalized Fra-1 gene expression in gastric cancer was upregulated 2.99 -fold $(\mathrm{P}<0.01)$. Similar tendency was seen by western blot experiments. IHC showed a similar pattern in protein expression with $\mathrm{qPCR}$ results and western blot results.
Adiseshaiah et al (2) found that FRA-1 was frequently overexpressed in squamous cell carcinomas of the stomach and esophagus. High Fra-1 expression is associated with a more malignant cell phenotype and Fra-1 could have a pivotal role in breast cancer progression (31). Philips et al (32) found that high Fra-1 concentration was crucial for the negative regulation of AP-1 activity by estradiol and that Fra-1 might take part in estradiol-induced inhibition of cell proliferation in ER alpha-breast cancer cells transfected with ER alpha expression construct. The results of the study by Shirsat et al (33) showed that overexpression of Fra-1 could lead to proliferation inhibition of C6 glioma cells. Our results are consistent with these reports.

Our results showed that Fra-1 was able to inhibit cell apoptosis of gastric cancer cell and increase the rate of $S$ phase of cell cycle. Deregulation of the cell cycle underlies the aberrant cell proliferation that characterizes cancer and loss of cell cycle checkpoint control promotes genetic instability. Moreover, we identified and confirmed that Fra-1 affected the expression level of CTTN and EZR in vitro by LC-MS/MS analyses. Our results suggested that Fra-1 might function by regulating the expression of CTTN and EZR. Zhang and Qi (34) found that MTSS1 suppressed cell migration and invasion by targeting CTTN in glioblastoma. Cortactin (CTTN) overexpression in osteosarcoma is correlated with its advanced stage and reduced survival (35). Luo et al (36) found that amplification and overexpression of CTTN (EMS1) contributed to the metastasis of esophageal squamous cell carcinoma by promoting cell migration and anoikis resistance. Our results also hinted that CTTN might play an important role in the malignancy.

The PI3K/Akt pathway is known to play key roles in cell apoptosis, cell proliferation, and cell survival in various kinds of cells (37). It is generally demonstrated that the PI3K/AKT signaling pathways regulate metastasis in a variety of cancer 
cells $(38,39)$. Our results showed that Fra-1 was correlated with dysregulation of PI3K/Akt and p53 signaling pathway in gastric cancer tissues in vitro and that Fra-1 overexpression affected the expression of PI3K, Akt, MDM2 and p53 in vivo. Degradation of p53 is regulated by its interaction with specific E3 ubiquitin ligases, the best known, being encoded by MDM2 (40). Damage to p53-dependent mechanism is often caused by overexpression of MDM2, which codes for a p53-regulating protein (41).

In summary, our results suggested that Fra-1 was upregulated in gastric cancer and affected the expression of CTTN and EZR in AGS gastric cancer cells. We found that Fra-1 was involved with dysregulation of PI3K/Akt and p53 signaling pathway in vitro and that Fra-1 overexpression affected the expression of PI3K, Akt, MDM2 and $\mathrm{p} 53$ in vivo. Thus, as shown (Fig. 6) we inferred that Fra-1 functions by regulating the expression of CCTN and EZR and dysactivation of the $\mathrm{PI} 3 \mathrm{~K} / \mathrm{Akt}$ and p53 signaling pathway.

\section{Acknowledgements}

The present study was supported by the National Natural Science Foundation of China (81272975, 81172302, 81402270 and 81402307); the Key Project of Hunan Provincial Natural Science Foundation (12JJ2044); the Project of Hunan Provincial Natural Science Foundation (12JJ3121); the Project of Hunan Provincial Development and Reform Commission; the Planned Science and Technology Project of Hunan Province (2010FJ3088 and 2012FJ2014).

\section{References}

1. Lu D, Chen S, Tan X, Li N, Liu C, Li Z, Liu Z, Stupack DG, Reisfeld RA and Xiang R: Fra-1 promotes breast cancer chemosensitivity by driving cancer stem cells from dormancy. Cancer Res 72: 3451-3456, 2012.

2. Adiseshaiah P, Lindner DJ, Kalvakolanu DV and Reddy SP: FRA-1 proto-oncogene induces lung epithelial cell invasion and anchorage-independent growth in vitro, but is insufficient to promote tumor growth in vivo. Cancer Res 67: 6204-6211, 2007.

3. Young MR and Colburn NH: Fra-1 a target for cancer prevention or intervention. Gene 379: 1-11, 2006.

4. Xiao S, Zhou Y, Yi W, Luo G, Jiang B, Tian Q, Li Y and Xue M: Fra-1 is downregulated in cervical cancer tissues and promotes cervical cancer cell apoptosis by p53 signaling pathway in vitro. Int J Oncol 46: 1677-1684, 2015.

5. Liu H, Ren G, Wang T, Chen Y, Gong C, Bai Y, Wang B, Qi H, Shen J, Zhu L, et al: Aberrantly expressed Fra-1 by IL-6/STAT3 transactivation promotes colorectal cancer aggressiveness through epithelial-mesenchymal transition. Carcinogenesis 36 459-468, 2015.

6. Wang HL, Wang J, Xiao SY, Haydon R, Stoiber D, He TC, Bissonnette $\mathrm{M}$ and Hart $\mathrm{J}$ : Elevated protein expression of cyclin D1 and Fra-1 but decreased expression of c-Myc in human colorectal adenocarcinomas overexpressing beta-catenin. Int $\mathrm{J}$ Cancer 101: 301-310, 2002.

7. Oliveira-Ferrer L, Kürschner M, Labitzky V, Wicklein D, Müller V, Lüers G, Schumacher U, Milde-Langosch K and Schröder C: Prognostic impact of transcription factor Fra-1 in ER-positive breast cancer: Contribution to a metastatic phenotype through modulation of tumor cell adhesive properties. J Cancer Res Clin Oncol: Feb 10, 2015 (Epub ahead of print).

8. Belguise K, Milord S, Galtier F, Moquet-Torcy G, Piechaczyk M and Chalbos D: The PKC $\theta$ pathway participates in the aberrant accumulation of Fra-1 protein in invasive ER-negative breast cancer cells. Oncogene 31: 4889-4897, 2012.

9. Davis WJ, Lehmann PZ and Li W: Nuclear PI3K signaling in cell growth and tumorigenesis. Front Cell Dev Biol 3: 24, 2015.

10. Brown KK and Toker A: The phosphoinositide 3-kinase pathway and therapy resistance in cancer. F1000Prime Rep 7: 13, 2015.
11. Zheng D, Zhu G, Liao S, Yi W, Luo G, He J, Pei Z, Li G and Zhou Y: Dysregulation of the PI3K/Akt signaling pathway affects cell cycle and apoptosis of side population cells in nasopharyngeal carcinoma. Oncol Lett 10: 182-188, 2015.

12. Liao S, Xiao S, Zhu G, Zheng D, He J, Pei Z, Li G and Zhou Y: CD38 is highly expressed and affects the PI3K/Akt signaling pathway in cervical cancer. Oncol Rep 32: 2703-2709, 2014.

13. Saha T, Kar RK and Sa G: Structural and sequential context of p53: A review of experimental and theoretical evidence. Prog Biophys Mol Biol 117: 250-263, 2015.

14. Meng X, Franklin DA, Dong J and Zhang Y: MDM2-p53 pathway in hepatocellular carcinoma. Cancer Res 74: 7161-7167, 2014.

15. Zhang Q, Zeng SX and Lu H: Targeting p53-MDM2-MDMX loop for cancer therapy. Subcell Biochem 85: 281-319, 2014.

16. Briest $F$ and Grabowski P: The p53 network as therapeutic target in gastroenteropancreatic neuroendocrine neoplasms. Cancer Treat Rev 41: 423-430, 2015.

17. Abraham AG and O'Neill E: PI3K/Akt-mediated regulation of p53 in cancer. Biochem Soc Trans 42: 798-803, 2014.

18. Livak KJ and Schmittgen TD: Analysis of relative gene expression data using real-time quantitative PCR and the 2(-Delta Delta C(T)) method. Methods 25: 402-408, 2001.

19. Zhou Y, Wang W, Zheng D, Peng S, Xiong W, Ma J, Zeng Z, Wu M, Zhou M, Xiang J, et al: Risk of nasopharyngeal carcinoma associated with polymorphic lactotransferrin haplotypes. Med Oncol 29: 1456-1462, 2012.

20. Zheng D, Liao S, Zhu G, Luo G, Xiao S, He J, Pei Z, Li G and Zhou Y: CD38 is a putative functional marker for side population cells in human nasopharyngeal carcinoma Cell Lines. Mol Carcinog: Jan 28, 2015 (Epub ahead of print). doi: 10.1002/ mc. 22279 .

21. Zhu W, Li J, Su J, Li J, Li J, Deng B, Shi Q, Zhou Y and Chen X: FOS-like antigen 1 is highly expressed in human psoriasis tissues and promotes the growth of HaCaT cells in vitro. Mol Med Rep 10: 2489-2494, 2014.

22. Hara A and Okayasu I: Cyclooxygenase- 2 and inducible nitric oxide synthase expression in human astrocytic gliomas: Correlation with angiogenesis and prognostic significance. Acta Neuropathol 108: 43-48, 2004.

23. Heo S, Spoerk S, Birner-Gruenberger R and Lubec G: Gel-based mass spectrometric analysis of hippocampal transmembrane proteins using high resolution LTQ Orbitrap Velos Pro. Proteomics 14: 2084-2088, 2014.

24. Haddad T and Kümmerer K: Characterization of photo-transformation products of the antibiotic drug Ciprofloxacin with liquid chromatography-tandem mass spectrometry in combination with accurate mass determination using an LTQ-Orbitrap. Chemosphere 115: 40-46, 2014.

25. Zhang JY, Wang F, Zhang H, Lu JQ and Qiao YJ: Rapid identification of polymethoxylated flavonoids in traditional Chinese medicines with a practical strategy of stepwise mass defect filtering coupled to diagnostic product ions analysis based on a hybrid LTQ-Orbitrap mass spectrometer. Phytochem Anal 25: 405-414, 2014

26. Carcas LP: Gastric cancer review. J Carcinog 13: 14, 2014.

27. Jemal A, Bray F, Center MM, Ferlay J, Ward E and Forman D: Global cancer statistics. CA Cancer J Clin 61: 69-90, 2011.

28. Choi B, Lee EJ, Park YS, Kim SM, Kim EY, Song Y, Kang SW, Rhu MH and Chang EJ: Pentraxin-3 silencing suppresses gastric cancer-related inflammation by inhibiting chemotactic migration of macrophages. Anticancer Res 35: 2663-2668, 2015.

29. Saavedra K, Valbuena J, Olivares W, Marchant MJ, Rodríguez A, Torres-Estay V, Carrasco-Avino G, Guzmán L, Aguayo F, Roa JC, et al: Loss of expression of reprimo, a p53-induced cell cycle arrest gene, correlates with invasive stage of tumor progression and p73 expression in gastric cancer. PLoS One 10: e0125834, 2015.

30. Praud D, Parpinel M, Serafini M, Bellocco R, Tavani A, Lagiou P, La Vecchia C and Rossi M: Non-enzymatic antioxidant capacity and risk of gastric cancer. Cancer Epidemiol 39: 340-345, 2015.

31. Belguise K, Kersual N, Galtier F and Chalbos D: FRA-1 expression level regulates proliferation and invasiveness of breast cancer cells. Oncogene 24: 1434-1444, 2005.

32. Philips A, Teyssier C, Galtier F, Rivier-Covas C, Rey JM, Rochefort $\mathrm{H}$ and Chalbos D: FRA-1 expression level modulates regulation of activator protein-1 activity by estradiol in breast cancer cells. Mol Endocrinol 12: 973-985, 1998.

33. Shirsat NV and Shaikh SA: Overexpression of the immediate early gene fra-1 inhibits proliferation, induces apoptosis, and reduces tumourigenicity of c6 glioma cells. Exp Cell Res 291: 91-100, 2003. 
34. Zhang S and Qi Q: MTSS1 suppresses cell migration and invasion by targeting CTTN in glioblastoma. J Neurooncol 121: 425-431, 2015.

35. Folio C,Zalacain M,Zandueta C, Ormazábal C, Sierrasesúmaga L, San Julián M, de las Rivas J, Toledo G, Lecanda F and PatiñoGarcía A: Cortactin (CTTN) overexpression in osteosarcoma correlates with advanced stage and reduced survival. Cancer Biomark 10: 35-41, 2011-2012.

36. Luo ML, Shen XM, Zhang Y, Wei F, Xu X, Cai Y, Zhang X, Sun YT, Zhan QM, Wu M, et al: Amplification and overexpression of CTTN (EMS1) contribute to the metastasis of esophageal squamous cell carcinoma by promoting cell migration and anoikis resistance. Cancer Res 66: 11690-11699, 2006.
37. Qiao M, Sheng S and Pardee AB: Metastasis and AKT activation. Cell Cycle 7: 2991-2996, 2008.

38. Vivanco I and Sawyers CL: The phosphatidylinositol 3-Kinase AKT pathway in human cancer. Nat Rev Cancer 2: 489-501, 2002.

39. Wagner EF and Nebreda AR: Signal integration by JNK and p38 MAPK pathways in cancer development. Nat Rev Cancer 9: 537-549, 2009

40. Chumakov PM: Function of the p53 gene: Choice between life and death. Biochemistry (Mosc) 65: 28-40, 2000.

41. Chipuk JE and Green DR: Dissecting p53-dependent apoptosis. Cell Death Differ 13: 994-1002, 2006. 